synchrotron radiation beamtime.

[1] Palmieri G. et al. PLoS ONE 6(10):e25888 (2011)[2] Menyhard D.K. et al. J Biol Chem 288 (24):17884-94 (2013)

Keywords: acylaminoacyl-peptidase, multimerization, substrate size selection

\section{MS6-P2 Structural characterization of dihydroorotase domain in yeast CAD multienzyme} Yujung Jeon ${ }^{1,2}$, Ian Ross ${ }^{2}$, Michael Landsberg $^{1}$, Ben Schultz
Hankamer ${ }^{2}$, Bostjan Kobe

1. School of Chemistry and Molecular Biosciences, The University
of Queensland
2. Institute of Molecular Biosciences, The University of
Queensland

email: yujung.jeon@uqconnect.edu.au

Carbamoyl phosphate synthetase II (CPS II), aspartate transcarbamoylase (ATC), and dihydroorotase (DHO) are three enzymes involved in the first three steps of de novo pyrimidine biosynthesis. In bacteria, these proteins are present in individual enzymes and function independently; however, in eukaryotes they are often found in a covalently linked form. In mammals, the enzymes are present as a large, multienzyme complex called CAD (CPS II-ATC-DHO, $243 \mathrm{kDa}$ ) and this complex has been proposed to form a homo-hexamer of $\sim 1.5 \mathrm{MDa}$ in the cellular environment. It is known that over-expression of CAD is required for proliferation and is associated with tumour cell development; thus, understanding the structural basis of the complex will be useful in cancer biology.

CAD in yeast, called URA2, is a bifunctional protein that has CPS II and ATC activity only. The DHO domain is substituted with pseudo-DHO amino acids sequence, which has lost its enzyme activity. The separately translated active DHO (URA4) compensates this loss of function, and this is homologous to bacterial type II DHO. This suggests the sequential gene duplication and fusion events occurred for CAD; thus, studying yeast URA2 may help understanding the molecular evolution. Crystal structure of the pseudo-DHO domain was determined at high resolution (1.2 §) and biochemical studies such as enzyme assay, MALS, and SAXS were performed. The protein presents in equilibrium between dimer and hexamer in solution, and this suggests its potential role of the domain in hexamerisation of the CAD complex in cellular environment despite its inactivity.

Keywords: enzyme 